\title{
Construction of the influenza A virus transmission tree in a college-based population: co-transmission and interactions between influenza A viruses
}

\author{
Xu-Sheng Zhang ${ }^{1,2^{*}}$ and Daniela De Angelis ${ }^{1,3}$
}

\begin{abstract}
Background: Co-infection of different influenza A viruses is known to occur but how viruses interact within co-infection remains unknown. An outbreak in a college campus during the 2009 pandemic involved two subtypes of influenza A: persons infected with pandemic A/H1N1; persons infected with seasonal A/H3N2 viruses; and persons infected with both at the same time (co-infection). This provides data to analyse the possible interaction between influenza A viruses within co-infection.

Methods: We extend a statistical inference method designed for outbreaks caused by one virus to that caused by two viruses. The method uses knowledge of which subtype each case is infected with (and whether they were co-infected), contact information and symptom onset date of each case in the influenza outbreak. We then apply it to construct the most likely transmission tree during the outbreak in the college campus.
\end{abstract}

Results: Analysis of the constructed transmission tree shows that the simultaneous presence of the two influenza viruses increases the infectivity and the transmissibility of $\mathrm{A} / \mathrm{H} 1 \mathrm{~N} 1$ virus but whether it changes the infectivity of $\mathrm{A} / \mathrm{H} 3 \mathrm{~N} 2$ is unclear. The estimation also shows that co-transmission of both subtypes from co-infection is low and therefore co-infection cannot be sustained on its own.

Conclusions: This study suggests that influenza A viruses within co-infected patients can interact in some ways rather than transmit independently, and this can enhance the spread of influenza A virus infection.

Keywords: Co-infection, Co-transmission, Inference, Influenza, Outbreak, Strain interactions

\section{Background}

Co-circulation of multiple types and subtypes of influenza virus has been commonly observed in human populations $[1,2]$. With advanced molecular techniques $[3,4]$, it is now becoming possible to rapidly detect different subtypes or strains of a disease agent within infected patients. There is accumulating evidence to show that the phenomenon that multiple subtypes of influenza A virus infect an individual simultaneously (i.e. co-infection) is

\footnotetext{
* Correspondence: xu-sheng.zhang@phe.gov.uk

${ }^{1}$ Centre for Infectious Disease Surveillance and Control, Public Health England, 61 Colindale Avenue, London NW9 5EQ, UK

${ }^{2}$ Medical Research Council Centre for Outbreak Analysis and Modelling, Department of Infectious Disease Epidemiology, Imperial College School of Public Health, Norfolk Place, London W2 1PG, UK

Full list of author information is available at the end of the article
}

not as rare as we previously thought [5-10]. Hence it is interesting to know how the simultaneous presence of two strains alters, compared to singly infected individuals, the transmissibility of each subtype and of both subtypes together. Some observations of transmission involved with co-infected individuals have been reported [11, 12]. One important observation reported is individuals co-infected with pandemic $\mathrm{A} / \mathrm{H} 1 \mathrm{~N} 1$ and seasonal $\mathrm{A} / \mathrm{H} 3 \mathrm{~N} 2$ influenza viruses within one outbreak at a college campus in Beijing, China during the 2009 pandemic [12]. Liu et al. [12] provides direct evidence that individuals co-infected with different subtypes can transmit viruses separately or simultaneously and provides detailed data for us to quantify the interactions between virus strains. The transmission characteristics can be directly estimated from the 
transmission tree; however, the difficulty for constructing the transmission tree is that some contacts were missed. Fortunately, recent development in statistical inference $[13,14]$ allows us to construct the transmission tree of a single influenza A virus from such a partial contact network. In this short report we extend this inference method to construct the transmission tree that includes two influenza A viruses and their co-infection. From the constructed tree we estimate the impact of co-infection on transmission. We use two parameters to characterise the impact: the co-transmission rate and an interaction parameter. Here we define the former as the rate at which two strains simultaneously transmit from doubly infected individuals to susceptible individuals; and the latter as the ratio of transmissibility of each single virus from coinfected individuals to the transmissibility of each single virus from singly infected individuals.

\section{Data}

Here we briefly summarize the data collection method and data of Liu et al. [12]. Investigations were conducted on all influenza like illness (ILI) cases identified during the outbreak. Epidemiological, clinical and contact tracing data were collected by interviewing patients and retrieving medical records. Viruses were identified by reverse-transcription polymerase chain reaction assays followed by sequence analysis. The heamagglutination inhibition tests were used to detect antibodies to both viruses. The outbreak is reported to have taken place within three buildings (two dormitories and one college clinic). No other cases at the college were reported. Buildings 1 and 2 (with a total membership of 235 and 191 persons, respectively) are next to each other and there is restricted access between the two and to the wider community. Forty five ILI cases were reported from 31 August to 10 September and forty $(N=40)$ had laboratory-confirmed influenza A infection. Three different types of infection were reported: 22 infected with pandemic $\mathrm{A} / \mathrm{H} 1 \mathrm{~N} 1$ virus, 12 infected with seasonal $\mathrm{A} /$ $\mathrm{H} 3 \mathrm{~N} 2$ virus and six co-infected with both influenza A viruses. In their sequences no substantial differences were observed between patients with mixed and single infections in either pandemic $\mathrm{A} / \mathrm{H} 1 \mathrm{~N} 1$ or seasonal $\mathrm{A} / \mathrm{H} 3 \mathrm{~N} 2$ virus. The clinical features were similar for patients with different infections and the six co-infected patients showed no more severe symptoms than the singly infected patients. Contacts between infected people are shown in Fig. 2 of Liu et al. [12] but this only extends to the contact network within one dormitory. The 'index' case with pandemic influenza A/H1N1 infection was a college student whose symptoms first occurred on 31 August, 2 days after his returning to college. Except for the index case, all patients with $\mathrm{A} / \mathrm{H} 1 \mathrm{~N} 1$ infection had not left the campus during the previous week. In contrast, the source of seasonal $\mathrm{A} / \mathrm{H} 3 \mathrm{~N} 2$ virus infection cannot be determined exactly although available data indicate that $\mathrm{A} / \mathrm{H} 3 \mathrm{~N} 2$ virus might have been prevailing in the college when the pandemic H1N1 virus was introduced. Before the isolation of cases and the initiation of prophylaxis among the campus population (5 September 2009), several patients visited the college clinic and the mixing between students of different dormitories was not frequent in comparison to the mixing between students within each dormitory.

\section{Methods}

For a fully traced transmission tree (i.e., the information of the infector $\mathbf{v}$ and time of symptom onset $t$ are collected), the infector $v(i)$ for each case $i$ (except the index case) and the duration between symptom onset of case $i$ and symptom onset of its infector $v(i): t_{i}-t_{v(i)}$ should be known. From these it is straightforward to estimate the generation interval distribution and transmissibility of infection. In reality, however, it is rare and difficult to record all the information. Based on the partially known contact tracing data and dates of symptom onset, Hens et al. [14] illustrated an inference method to reconstruct the most likely transmission tree that involved with only one virus. Here we further develop it to a transmission tree during an outbreak that involves with two viruses of similar epidemiological characteristics.

In general, a possible transmission tree can be described by $p_{i j}(\mathbf{v}, \mathbf{w}, \boldsymbol{\varphi} ; \boldsymbol{\theta})$, the probability that case $j$ is the infector of case $i$, given the duration between symptom onset of case $i$ and case $j$, given the information on the possible infector $\mathbf{v}$ and the known contacts $\mathbf{w}$, and given the types $\phi$ of infection of both cases. Following Hens et al [14], its total log-likelihood is given by:

$$
L(\boldsymbol{\theta} \mid t, \mathbf{v}, \mathbf{w}, \boldsymbol{\phi})=\sum_{i=2}^{N} \sum_{j=1}^{N} p_{i j}(\mathbf{v}, \mathbf{w}, \boldsymbol{\phi} ; \boldsymbol{\theta}) \log \left[g\left(t_{i}-t_{j} \mid \boldsymbol{\theta}\right)\right]
$$

The sum runs through all the non-zero $p_{i j}$. Here $\mathrm{g}(\Delta t \mid \boldsymbol{\theta})$ denotes the probability density of the generation interval distribution of influenza infection, with $\boldsymbol{\theta}$ representing the set of parameters that characterise the probability density distribution. Different distributions such as gamma, lognormal and Weibull can be used to describe the distribution of generation intervals [14]. Here we assume it follows a Weibull distribution:

$$
\begin{aligned}
& g(\Delta t \mid \boldsymbol{\theta})=\frac{b}{a}\left(\frac{\Delta t}{a}\right)^{b-1} \exp \left(-\left(\frac{\Delta t}{a}\right)^{b}\right) \text { when } \Delta t \geq 0, \\
& g(\Delta t \mid \boldsymbol{\theta})=0 \text { otherwise. }
\end{aligned}
$$

The distribution has two parameters (i.e., $\boldsymbol{\theta}=\{a, b\}$ ): scale parameter $a$ and shape parameter $b$, such that the mean is 
$T=a \Gamma(1+1 / b)$ and the variance is $\sigma^{2}=a^{2}[\Gamma(1+2 / b)-\Gamma(1+$ $\left.1 / b)^{2}\right]$. Here $\Gamma()$ is the Gamma function.

Compared with outbreaks that involved one virus and one transmission process [14], this outbreak [12] involved two viruses and five possible transmission processes: from $\mathrm{A} / \mathrm{H} 1 \mathrm{~N} 1$ to $\mathrm{A} / \mathrm{H} 1 \mathrm{~N} 1$; from $\mathrm{A} / \mathrm{H} 3 \mathrm{~N} 2$ to $\mathrm{A} / \mathrm{H} 3 \mathrm{~N} 2$; from coinfection to $\mathrm{A} / \mathrm{H} 1 \mathrm{~N} 1$; from co-infection to $\mathrm{A} / \mathrm{H} 3 \mathrm{~N} 2$ and from co-infection to co-infection. However, the data given in Liu et al. [12] only provide the relevant information for three transmission processes: from $\mathrm{A} / \mathrm{H} 1 \mathrm{~N} 1$ to $\mathrm{A} / \mathrm{H} 1 \mathrm{~N} 1$; from co-infection to $\mathrm{A} / \mathrm{H} 1 \mathrm{~N} 1$; and from co-infection to co-infection. The simple calculations show that the mean generation intervals (and their standard deviations) for the three transmission processes are $1.8(0.8), 1.2(0.4), 1.6$ (0.6) days, respectively. In this data there is no evidence that they differ. As no data is available for the transmission processes that were involved with $\mathrm{A} / \mathrm{H} 3 \mathrm{~N} 2$ [12], it is difficult to estimate their generation intervals and judge how they differ from those generation intervals involved with pandemic A/H1N1. Nevertheless, a recent systematic review [15] indicates that the generation interval of pandemic $\mathrm{A} / \mathrm{H} 1 \mathrm{~N} 1$ virus was similar to that of the seasonal flu. Further, as observed by Liu et al. [12], the three different types of infections have similar epidemiological characteristics. In view of these, the same generation interval distribution is assumed for the three types of infection.

The probability that case $i$ is infected by case $j, p_{i j}$, can be calculated as the probability of observing the duration between the symptom onsets in cases $i$ and $j, \mathrm{~g}\left(t_{\mathrm{i}}-t_{\mathrm{j}} \mid \boldsymbol{\theta}\right)$, times the probability of a potentially infectious contact between $i$ and $j, \pi_{i j}$, normalized by the probability of $i$ being infected by any other case $k$ :

$$
p_{i j}(\mathbf{v}, \mathbf{w}, \boldsymbol{\phi} ; \boldsymbol{\theta})=\frac{\pi_{i j}(\mathbf{v}, \mathbf{w}, \boldsymbol{\phi}) g\left(t_{i}-t_{j} \mid \boldsymbol{\theta}\right)}{\sum_{k \neq i} \pi_{i k}(\mathbf{v}, \mathbf{w}, \boldsymbol{\phi}) g\left(t_{i}-t_{k} \mid \boldsymbol{\theta}\right)}
$$

The probability of a potentially infectious contact between $i$ and $j, \pi_{i j}$, is based on the contact information $(\mathbf{v}, \mathbf{w})$ collected during the outbreak and the types $\boldsymbol{\phi}$ of infection of both cases $i$ and $j$. To distinguish different types of infection and to reflect the fact that there is only limited mixing between students in building 1 and building 2, we define the following,

$\psi_{i j}=1$ if case $i$ and case $j$ reside in the same dormitory and are the same type of infection or if case $j$ is a co-infection; $\psi_{i j}=w$ if case $i$ and case $j$ reside in different dormitories and are the same type of infection or if case $j$ is a co-infection;

$\psi_{i j}=0$ otherwise.

The variable $w$ defines the extent of contact rates between students in two dormitories in relation to contact rates within dormitories: $w=0$ implies that contacts between dormitories are forbidden and $w=1$ suggests that contacts between dormitories are the same as that within each dormitory. Student access to the college clinic was not restricted. The original numberings of patients in Fig. 2 of Liu et al. [12] are given in accordance with the order of symptom onset within each building. For the convenience of our analysis, the forty patients have been re-indexed in the order of symptom onset as $1,2, \ldots, 40$ (see Fig. 1). If cases $i$ and $j$ form a likely transmission pair (i.e., $v(i)=j$ ) and there is only one possible infector, $\pi_{i j}(\mathbf{v}, \mathbf{w}, \boldsymbol{\phi})=\psi_{i j}$ and $p_{i j}(\mathbf{v}, \mathbf{w}, \boldsymbol{\phi} ; \boldsymbol{\theta})=1$. If the outbreak investigation reveals that case $i$ is not the index case and does not contact any of the $i-1$ cases that developed symptoms before case $i$, the probability of a potentially infectious contact is $\pi_{i j}(\mathbf{v}, \mathbf{w}, \boldsymbol{\phi})=\psi_{i j} / \eta_{i-1}$ Here $\eta_{i-1}$ is the 'effective' number of infections that developed symptoms before case $i$ and are of the same type of infection as case $i$ or co-infection, and is calculated as $\eta_{i-1}=\sum_{k=1}^{i-1} \psi_{i k}$.

Given the contact information $(\mathbf{v}, \mathbf{w})$, the most likely transmission tree can be obtained by finding the values of parameters $\boldsymbol{\theta}=\{a, b\}$ that maximize the total loglikelihood (1). The downhill simplex method [16] was used to locate the maximum-likelihood estimate (MLE), $\hat{\boldsymbol{\theta}}$, of the parameters. We also used the MCMC method to sample the values of parameters $\boldsymbol{\theta}$ and find their medians, 2.5 percentile and 97.5 percentile.

From the probabilities that case $i$ is infected by case $j$ : $\left\{p_{1 i}, \ldots, p_{N i}\right\}$, we can sample a transmission tree in which all cases are connected and case 1 is the index case (Fig. 1) and further estimate transmissibility. The case reproduction number is the average number of secondary cases generated by primary cases, which measures the transmissibility of infection. The overall casereproduction number on day $t, R_{t}$, can be estimated by summing over all these infectious contacts:

$$
R_{t}=\sum_{j} \sum_{i=2} p_{i j}(\mathbf{v}, \mathbf{w}, \boldsymbol{\phi} ; \hat{\boldsymbol{\theta}})
$$

We can further estimate the infection type-specific reproductive numbers when both case $i$ and case $j$ are of the same type of infection:

$$
\begin{aligned}
& R_{\mathrm{e}}^{1}=\sum_{j} \sum_{i=2} p_{i j}(\mathbf{v}, \mathbf{w}, \boldsymbol{\phi} ; \hat{\boldsymbol{\theta}}) \delta_{i j ; \mathrm{H} 1 \mathrm{~N} 1} \\
& R_{\mathrm{e}}^{2}=\sum_{j} \sum_{i=2} p_{i j}(\mathbf{v}, \mathbf{w}, \boldsymbol{\phi} ; \hat{\boldsymbol{\theta}}) \delta_{i j ; \mathrm{H} 3 \mathrm{~N} 2} \\
& R_{\mathrm{e}}^{\mathrm{C}}=\sum_{j} \sum_{i=2} p_{i j}(\mathbf{v}, \mathbf{w}, \boldsymbol{\phi} ; \hat{\boldsymbol{\theta}}) \delta_{i j ; \text { co infection }}
\end{aligned}
$$

Here the symbol $\delta_{i j, \mathrm{TYPE}}$ is defined as: $\delta_{i j, \mathrm{TYPE}}=1$ if both cases $i$ and $j$ are of the infection TYPE; $\delta_{i j \text { TYPE }}=0$ 


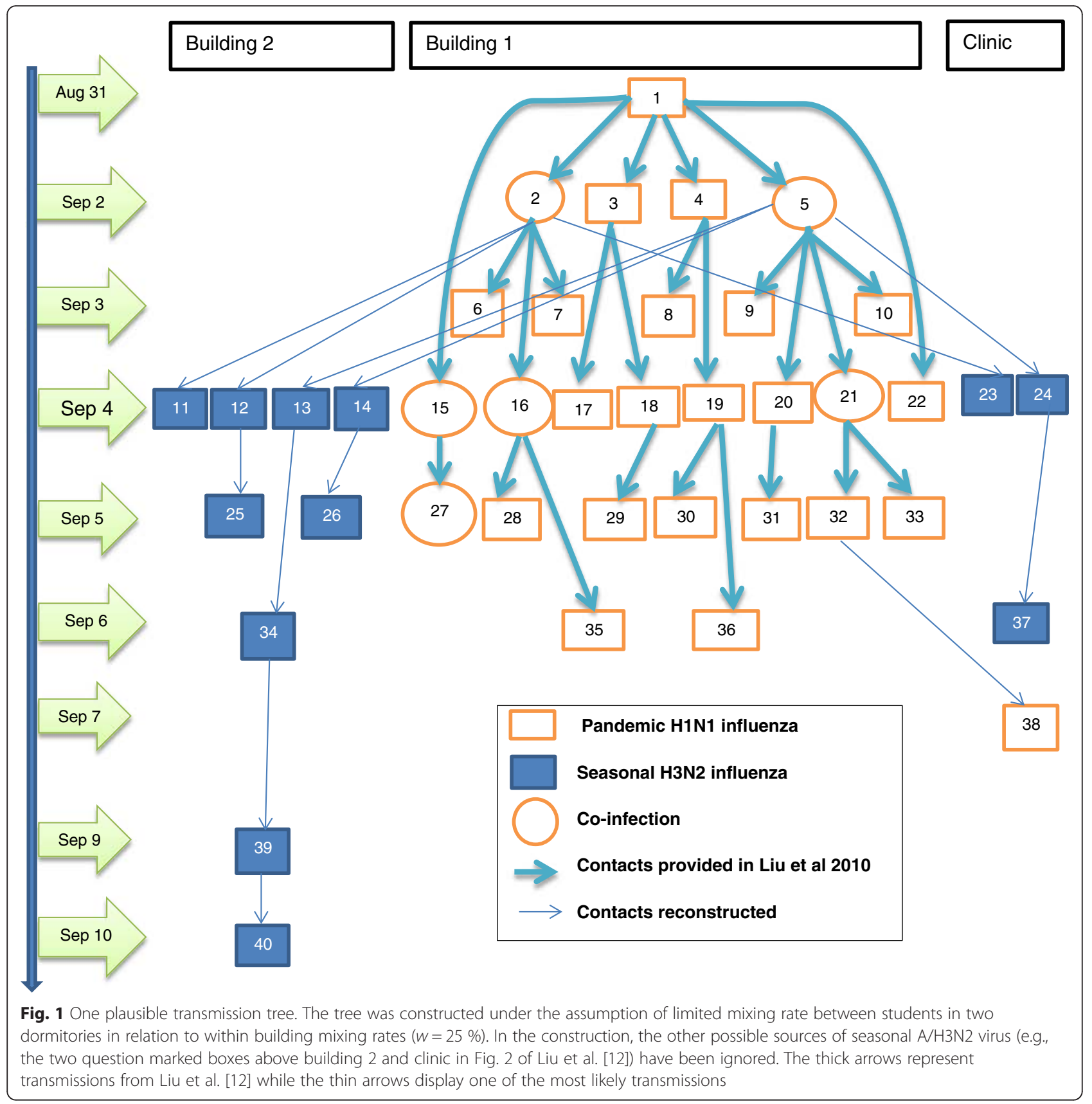

otherwise. To characterise the interaction between two types of virus within co-infected individuals, we further estimate the reproductive numbers of $\mathrm{A} / \mathrm{H} 1 \mathrm{~N} 1$ and $\mathrm{A} /$ H3N2 due to co-infected individuals as:

$$
\begin{aligned}
& R_{\mathrm{e}, \mathrm{C}}^{1}=\sum_{j} \sum_{i=2} p_{i j}(\mathbf{v}, \mathbf{w}, \boldsymbol{\phi} ; \hat{\boldsymbol{\theta}}) \Delta_{i j ; \mathrm{H} 1 \mathrm{~N} 1} \\
& R_{\mathrm{e}, \mathrm{C}}^{2}=\sum_{j} \sum_{i=2} p_{i j}(\mathbf{v}, \mathbf{w}, \boldsymbol{\phi} ; \hat{\boldsymbol{\theta}}) \Delta_{i j ; \mathrm{H} 3 \mathrm{~N} 2}
\end{aligned}
$$

Here $\Delta_{i j \text { TYPE }}$ is defined as: $\Delta_{i j, \mathrm{TYPE}}=1$ if infector case $j$ is co-infected while the infectee case $i$ is singly infected with type TYPE; otherwise $\Delta_{i j \text { TYPE }}=0$. The strain interactions within co-infections are estimated as:

$$
\begin{aligned}
& \phi_{1}=\frac{R_{\mathrm{e}, \mathrm{C}}^{1}}{R_{\mathrm{e}}^{1}} \\
& \phi_{2}=\frac{R_{\mathrm{e}, \mathrm{C}}^{2}}{R_{\mathrm{e}}^{2}}
\end{aligned}
$$

Here $\phi_{1}$ measures the effect on infectivity of A/H1N1 within co-infections and $\phi_{2}$ measures the effect on infectivity of A/H3N2 within co-infections. 


\section{Ethics considerations}

Data that were used in the analysis of this study were extracted from a previous study [12] and hence did not require Human Resource Ethics committee approval.

\section{Results and discussion}

The data shown in Fig. 2 of Liu et al. [12] indicates that the index case is infected with pandemic $\mathrm{A} / \mathrm{H} 1 \mathrm{~N} 1$ virus. Among the six cases that the index case infects, three patients (i.e., case 2, 5, and 15) are co-infected with both $\mathrm{A} / \mathrm{H} 1 \mathrm{~N} 1$ and $\mathrm{A} / \mathrm{H} 3 \mathrm{~N} 2$. It was assumed that seasonal A/ H3N2 virus was endemic [12], however, the infectious contacts with $\mathrm{A} / \mathrm{H} 3 \mathrm{~N} 2$ of these three co-infected cases were unknown. Several possible scenarios are possible: cases 2,5 and 15 were exposed to pandemic A/H1N1 virus when they were still infectious with $\mathrm{A} / \mathrm{H} 3 \mathrm{~N} 2$; cases 2, 5 and 15 were further exposed to seasonal $\mathrm{A} / \mathrm{H} 3 \mathrm{~N} 2$ virus soon after they had acquired pandemic $\mathrm{A} / \mathrm{H} 1 \mathrm{~N} 1$ virus from the index case; the three cases were infected simultaneously with both $\mathrm{A} / \mathrm{H} 1 \mathrm{~N} 1$ and $\mathrm{A} / \mathrm{H} 3 \mathrm{~N} 2$ viruses from the index case who was actually co-infected before contact with cases 2, 5 and 15 but was incorrectly typed as a single infection with pandemic $\mathrm{A} / \mathrm{H} 1 \mathrm{~N} 1$ virus. For simplicity, we use all the contact information for cases within building 1 , especially the pathways from the index case to the three co-infections, ignoring the possible pathways for transmitting background endemic seasonal $\mathrm{A} / \mathrm{H} 3 \mathrm{~N} 2$ virus.

The maximum likelihood estimates of the infective contact probabilities $p_{i j}(\mathbf{v}, \mathbf{w}, \boldsymbol{\phi} ; \boldsymbol{\theta})$ are listed in Table 1 from which one sample transmission tree is shown in Fig. 1. The MLEs of the generation interval distribution
$g(\Delta t \mid \boldsymbol{\theta})$ and the time course of the mean case reproductive number $R_{t}$ are shown in Fig. 2. The generation interval has a mean of 1.72 days and a standard deviation (SD) of 0.74 days. The mean case reproductive number over the whole outbreak is 1.57 with $\mathrm{SD}=0.022$. Before the isolation of cases and the initiation of prophylaxis among the campus population occurred (5 September 2009), the reproductive number is estimated to be 2.80 with $\mathrm{SD}=0.016$; after this it declines to 0.341 with $\mathrm{SD}=$ 0.047. This clearly shows the effectiveness of isolation and prophylaxis.

To measure the interactions between two influenza A viruses, we first estimated the values of infection typespecific reproductive numbers before the initiation of isolation and prophylaxis occurred. Because most transmissions that involve $\mathrm{A} / \mathrm{H} 1 \mathrm{~N} 1$ and co-infections are known, the estimates of the infection type-specific reproductive numbers are stable at $R_{e}^{1}=1.44$ and $R_{e}^{C}=$ 0.67 , and the reproductive number of $\mathrm{A} / \mathrm{H} 1 \mathrm{~N} 1$ due to co-infection is $R_{e, C}^{1}=2.01$. In contrast, transmissions that involve $\mathrm{A} / \mathrm{H} 3 \mathrm{~N} 2$ were missing and so estimates of their reproductive numbers show some uncertainty: $R_{e}^{2}$ has a mean 0.48 and $95 \%$ confidence interval of [0.45, 0.51], and the reproductive number of $\mathrm{A} / \mathrm{H} 3 \mathrm{~N} 2$ due to coinfection is $R_{e, C}^{2}=1.60[1.58,1.62]$. Although the estimate of $R_{e}^{1}$ for $\mathrm{A} / \mathrm{H} 1 \mathrm{~N} 1$ is in agreement with the usual estimates [15, 17], that of $R_{e}^{2}$ for $\mathrm{A} / \mathrm{H} 3 \mathrm{~N} 2$ is much smaller than other estimates which range from 1.19 to 1.37 [17]. The likely reason for this difference lies in the fact that pandemic $\mathrm{A} / \mathrm{H} 1 \mathrm{~N} 1$ is a novel virus while $\mathrm{A} /$ $\mathrm{H} 3 \mathrm{~N} 2$ is an endemic seasonal virus in the study region [12] so some pre-existing immunity against A/H3N2
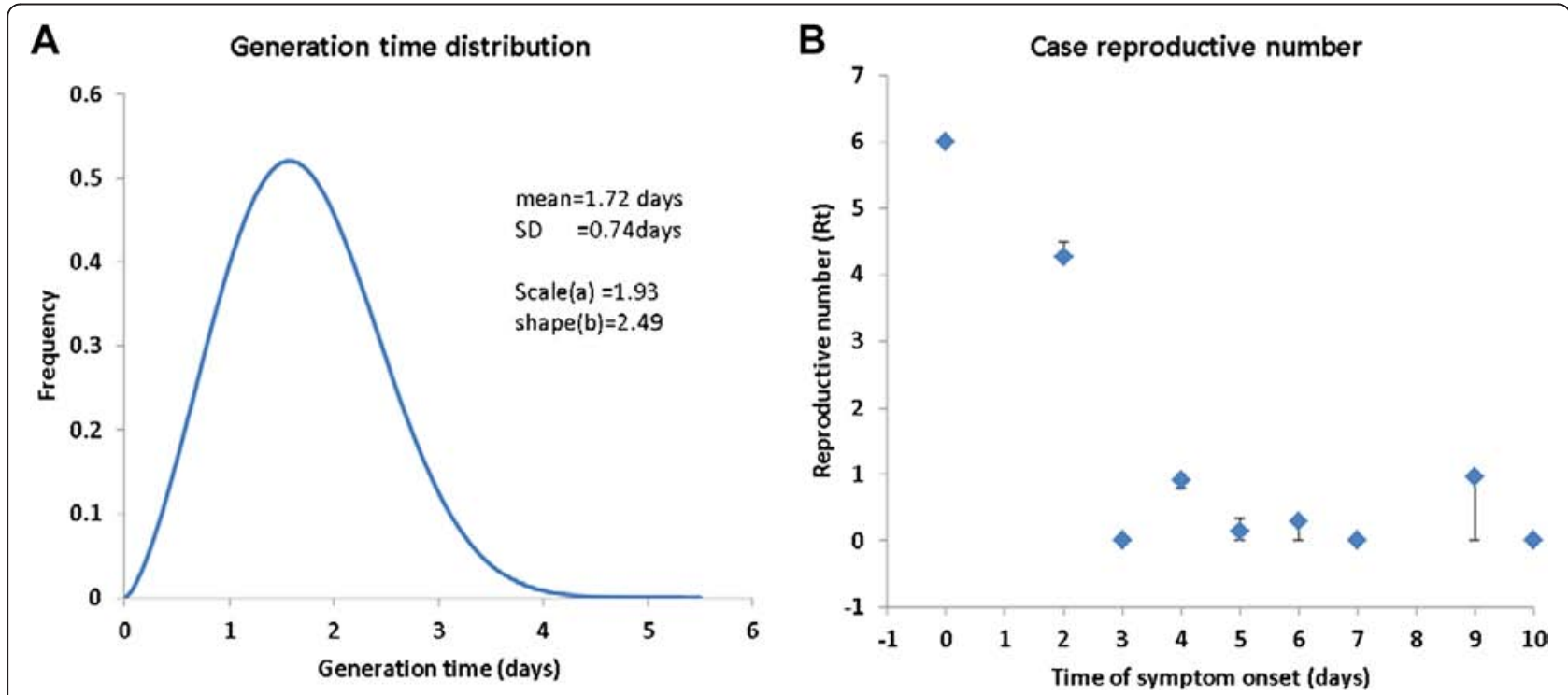

Fig. 2 Constructed transmission tree of influenza A virus: a The relative frequency of the generation intervals; b The average case reproduction number $R_{t}$ as it varies with time. Bars represent $95 \%$ nonparametric bootstrap percentile confidence intervals generated from one million possible transmission trees sampled from the contact probabilities listed in Table 1 
Table 1 The most likely contact probabilities under the limited mixing between dormitories ( $w=25 \%$ )

\begin{tabular}{|c|c|c|c|c|c|}
\hline Infectee $i$ & Infector $j$ & $p_{i j}$ & Infectee $i$ & Infector $j$ & $p_{i j}$ \\
\hline $1(1)$ & - & & $35(1)$ & $16(0)$ & 1.0 \\
\hline $2(0)$ & $1(1)^{*}$ & 1.0 & $36(1)$ & $19(1)$ & 1.0 \\
\hline $3(1)$ & $1(1)$ & 1.0 & $37(2)$ & $2(0)$ & 0.001602 \\
\hline $4(1)$ & $1(1)$ & 1.0 & $37(2)$ & $5(0)$ & 0.001602 \\
\hline $5(0)$ & $1(1)^{*}$ & 1.0 & $37(2)$ & $11(2)$ & 0.08588 \\
\hline $6(1)$ & $2(0)$ & 1.0 & $37(2)$ & $12(2)$ & 0.08588 \\
\hline $7(1)$ & $2(0)$ & 1.0 & $37(2)$ & $13(2)$ & 0.08588 \\
\hline $8(1)$ & $4(1)$ & 1.0 & $37(2)$ & $14(2)$ & 0.08588 \\
\hline $9(1)$ & $5(0)$ & 1.0 & $37(2)$ & $15(0)$ & 0.08588 \\
\hline $10(1)$ & $5(0)$ & 1.0 & $37(2)$ & $16(0)$ & 0.08588 \\
\hline $11(2)$ & $2(0)$ & 0.5 & $37(2)$ & $21(0)$ & 0.08588 \\
\hline $11(2)$ & $5(0)$ & 0.5 & $37(2)$ & $23(2)$ & 0.08588 \\
\hline $12(2)$ & $2(0)$ & 0.5 & $37(2)$ & $24(2)$ & 0.08588 \\
\hline $12(2)$ & $5(0)$ & 0.5 & $37(2)$ & $25(2)$ & 0.074625 \\
\hline $13(2)$ & $2(0)$ & 0.5 & $37(2)$ & $26(2)$ & 0.074625 \\
\hline $13(2)$ & $5(0)$ & 0.5 & $37(2)$ & $27(0)$ & 0.074625 \\
\hline $14(2)$ & $2(0)$ & 0.5 & $38(1)$ & $1(1)$ & 3.67E-11 \\
\hline $14(2)$ & $5(0)$ & 0.5 & $38(1)$ & $2(0)$ & 2.53E-05 \\
\hline $15(0)$ & $1(1)^{*}$ & 1.0 & $38(1)$ & $3(1)$ & 2.53E-05 \\
\hline $16(0)$ & $2(0)$ & 1.0 & $38(1)$ & $4(1)$ & 2.53E-05 \\
\hline $17(1)$ & $3(1)$ & 1.0 & $38(1)$ & $5(0)$ & 2.53E-05 \\
\hline $18(1)$ & $3(1)$ & 1.0 & $38(1)$ & $6(1)$ & 0.001691 \\
\hline $19(1)$ & $4(1)$ & 1.0 & $38(1)$ & $7(1)$ & 0.001691 \\
\hline $20(1)$ & $5(0)$ & 1.0 & $38(1)$ & $8(1)$ & 0.001691 \\
\hline $21(0)$ & $5(0)$ & 1.0 & $38(1)$ & $9(1)$ & 0.001691 \\
\hline $22(1)$ & $1(1)$ & 1.0 & $38(1)$ & $10(1)$ & 0.001691 \\
\hline $23(2)$ & $2(0)$ & 0.5 & $38(1)$ & $15(0)$ & 0.024945 \\
\hline $23(2)$ & $5(0)$ & 0.5 & $38(1)$ & $16(0)$ & 0.024945 \\
\hline $24(2)$ & $2(0)$ & 0.5 & $38(1)$ & $17(1)$ & 0.024945 \\
\hline $24(2)$ & $5(0)$ & 0.5 & $38(1)$ & $18(1)$ & 0.024945 \\
\hline $25(2)$ & $2(0)$ & 0.011463 & $38(1)$ & $19(1)$ & 0.024945 \\
\hline $25(2)$ & $5(0)$ & 0.011463 & $38(1)$ & $20(1)$ & 0.024945 \\
\hline $25(2)$ & $11(2)$ & 0.144752 & $38(1)$ & $21(0)$ & 0.024945 \\
\hline $25(2)$ & $12(2)$ & 0.144752 & $38(1)$ & $22(1)$ & 0.024945 \\
\hline $25(2)$ & $13(2)$ & 0.144752 & $38(1)$ & $27(0)$ & 0.090627 \\
\hline $25(2)$ & $14(2)$ & 0.144752 & $38(1)$ & $28(1)$ & 0.090627 \\
\hline $25(2)$ & $15(0)$ & 0.036188 & $38(1)$ & $29(1)$ & 0.090627 \\
\hline $25(2)$ & $16(0)$ & 0.036188 & $38(1)$ & $30(1)$ & 0.090627 \\
\hline $25(2)$ & $21(0)$ & 0.036188 & $38(1)$ & $31(1)$ & 0.090627 \\
\hline $25(2)$ & $23(2)$ & 0.144752 & $38(1)$ & $32(1)$ & 0.090627 \\
\hline $25(2)$ & $24(2)$ & 0.144752 & $38(1)$ & $33(1)$ & 0.090627 \\
\hline $26(2)$ & $2(0)$ & 0.011463 & $38(1)$ & $35(1)$ & 0.07875 \\
\hline $26(2)$ & $5(0)$ & 0.011463 & $38(1)$ & $36(1)$ & 0.07875 \\
\hline
\end{tabular}

Table 1 The most likely contact probabilities under the limited mixing between dormitories ( $w=25 \%$ ) (Continued)

\begin{tabular}{|c|c|c|c|c|c|}
\hline $26(2)$ & $11(2)$ & 0.144752 & $39(2)$ & $2(0)$ & 1.70E-10 \\
\hline $26(2)$ & $12(2)$ & 0.144752 & $39(2)$ & $5(0)$ & $1.70 \mathrm{E}-10$ \\
\hline $26(2)$ & $13(2)$ & 0.144752 & $39(2)$ & $11(2)$ & 0.00047 \\
\hline $26(2)$ & $14(2)$ & 0.144752 & $39(2)$ & $12(2)$ & 0.00047 \\
\hline $26(2)$ & $15(0)$ & 0.036188 & $39(2)$ & $13(2)$ & 0.00047 \\
\hline $26(2)$ & $16(0)$ & 0.036188 & $39(2)$ & $14(2)$ & 0.00047 \\
\hline $26(2)$ & $21(0)$ & 0.036188 & $39(2)$ & $15(0)$ & 0.000118 \\
\hline $26(2)$ & $23(2)$ & 0.144752 & $39(2)$ & $16(0)$ & 0.000118 \\
\hline $26(2)$ & $24(2)$ & 0.144752 & $39(2)$ & $21(0)$ & 0.000118 \\
\hline $27(0)$ & $15(0)$ & 1.0 & $39(2)$ & $23(2)$ & 0.00047 \\
\hline $28(1)$ & $15(0)$ & 0.5 & $39(2)$ & $24(2)$ & 0.00047 \\
\hline $28(1)$ & $16(0)$ & 0.5 & $39(2)$ & $25(2)$ & 0.031389 \\
\hline $29(1)$ & $16(0)$ & 0.5 & $39(2)$ & $26(2)$ & 0.031389 \\
\hline $29(1)$ & $16(0)$ & 0.5 & $39(2)$ & $27(2)$ & 0.007847 \\
\hline $30(1)$ & $19(1)$ & 1.0 & $39(2)$ & $34(2)$ & 0.4631 \\
\hline $31(1)$ & $19(1)$ & 0.5 & $39(2)$ & $37(2)$ & 0.4631 \\
\hline $31(1)$ & $20(1)$ & 0.5 & $40(2)$ & $2(0)$ & $8.39 \mathrm{E}-15$ \\
\hline $32(1)$ & $21(0)$ & 1.0 & $40(2)$ & $5(0)$ & 8.39E-15 \\
\hline $33(1)$ & $21(0)$ & 1.0 & $40(2)$ & $11(2)$ & 8.98E-07 \\
\hline 34 (2) & $2(0)$ & 0.000535 & $40(2)$ & $12(2)$ & 8.98E-07 \\
\hline $34(2)$ & $5(0)$ & 0.000535 & $40(2)$ & $13(2)$ & 8.98E-07 \\
\hline $34(2)$ & $11(2)$ & 0.114752 & $40(2)$ & $14(2)$ & 8.98E-07 \\
\hline $34(2)$ & $12(2)$ & 0.114752 & $40(2)$ & $15(0)$ & 2.24E-07 \\
\hline $34(2)$ & $13(2)$ & 0.114752 & $40(2)$ & $16(0)$ & 2.24E-07 \\
\hline $34(2)$ & $14(2)$ & 0.114752 & $40(2)$ & $21(0)$ & 2.24E-07 \\
\hline $34(2)$ & $15(0)$ & 0.028688 & $40(2)$ & $23(2)$ & 8.98E-07 \\
\hline $34(2)$ & $16(0)$ & 0.028688 & $40(2)$ & $24(2)$ & 8.98E-07 \\
\hline $34(2)$ & $21(0)$ & 0.028688 & $40(2)$ & $25(2)$ & 0.000308 \\
\hline 34 (2) & $23(2)$ & 0.114752 & $40(2)$ & $26(2)$ & 0.000308 \\
\hline $34(2)$ & $24(2)$ & 0.114752 & $40(2)$ & $27(0)$ & 7.71E-05 \\
\hline $34(2)$ & $25(2)$ & 0.099713 & $40(2)$ & $34(2)$ & 0.020572 \\
\hline $34(2)$ & $26(2)$ & 0.099713 & $40(2)$ & $37(2)$ & 0.020572 \\
\hline $34(2)$ & $27(0)$ & 0.024928 & $40(2)$ & $39(2)$ & 0.958157 \\
\hline
\end{tabular}

The numbers in the brackets are the type of infection: 0, 1, 2 representing co-infection, infection with pandemic $\mathrm{A} / \mathrm{H} 1 \mathrm{~N} 1$ virus alone and infection with seasonal $\mathrm{A} / \mathrm{H} 3 \mathrm{~N} 2$ virus alone, respectively. The three effective contacts from the index pandemic $\mathrm{A} / \mathrm{H} 1 \mathrm{~N} 1$ to co-infection that are marked with asterisk are as given by Liu et al. [12]. The maximum likelihood estimates of Weibull distribution parameters for the generation time are scale $=1.93$ and shape $=2.49$

due to the previous infections and/or vaccination reduces its susceptibility and hence its spread. In the presence of co-infection, infectivity of $\mathrm{A} / \mathrm{H} 1 \mathrm{~N} 1$ increases moderately with $\phi_{1}=1.4$. However, the increased infectivity of $\mathrm{A} / \mathrm{H} 3 \mathrm{~N} 2$ in the presence of co-infection is large: $\phi_{2}=3.28$ [95 \% CI: 3.18 3.38] and so co-infection is estimated to contribute greatly to the outbreak. This is not inconsistent with Liu et al. [12]'s observation that no 
patients had detectable hemagglutination inhibiting antibodies against pandemic H1N1 virus in their acutephase samples. Although the presence of co-infection can increase the infectivity of each component influenza virus, co-infection itself cannot spread successfully because the co-transmissibility measured by $R_{e}^{C}$ is estimated to be 0.67 , which is less than one.

The above results are obtained under the assumption of $w=25 \%$, which is used to reflect the observation although there were activities outside the dormitory, the members of the 2 buildings did not interact with each other to any significant extent (p1361, second column, Liu et al. [12]). If different values of $w$ are chosen, the estimation of transmissibility involved with $\mathrm{A} / \mathrm{H} 3 \mathrm{~N} 2$ virus changes although the results of the overall transmissibility and those involved with $\mathrm{A} / \mathrm{H} 1 \mathrm{~N} 1$ remain nearly the same. With a low value of $w$ (i.e. the mixing between two dormitories become more restrictive), the reproductive number of $\mathrm{A} / \mathrm{H} 3 \mathrm{~N} 2$ due to co-infection $\left(R_{e, C}^{2}\right)$ decreases while $R_{e}^{2}$ increases; this consequently reduces the value of $\phi_{2}$. For example, the estimate of $\phi_{2}$ becomes 3.00 [2.92, 3.13] when $w=0.1$. This is the outcome because without the index case of $\mathrm{A} / \mathrm{H} 3 \mathrm{~N} 2$ infection, the only possible infectors of $\mathrm{A} / \mathrm{H} 3 \mathrm{~N} 2$ cases $11,12,13,14$ in building 2 are coinfection cases 2 and 5, which is independent of the limited mixing rate between two dormitories $(w)$. In the situation without limitation in mixing rates between two dormitories (i.e., $w=1.0$ ), the estimate of $\phi_{2}$ is 4.57 [4.42, 4.70]. This result implies that in the absence of any unidentified index cases of $\mathrm{A} / \mathrm{H} 3 \mathrm{~N} 2$ infection, co-infection can enhance the transmissibility of each component virus.

However, all the cases reported in building 2 were infections with $\mathrm{A} / \mathrm{H} 3 \mathrm{~N} 2$ virus alone. In view of the restrictive mixing between two dormitories, it is likely that there is an unknown index case of $\mathrm{A} / \mathrm{H} 3 \mathrm{~N} 2$ infection within building 2 , although it was not reported. An analysis listed in the Additional file 1 shows that such a hidden index case could change the above conclusion: the reproductive number of $\mathrm{A} / \mathrm{H} 3 \mathrm{~N} 2$ due to co-infection $\left(R_{e, C}^{2}\right)$ might not exceed the reproductive number by its own $\left(R_{e}^{2}\right)$ and therefore co-infection could not enhance the infectivity of A/H3N2. Jombart et al. [18] have developed a Bayesian method to reconstruct disease outbreaks by combining epidemiological and genomic data. This may allow for the tests of whether co-infection cases in building 1 are the infectors of $\mathrm{A} / \mathrm{H} 3 \mathrm{~N} 2$ cases in building 2 or whether there is a hidden index case of A/ H3N2 infection in building 2. Unfortunately, the representative sequences deposited in GenBank by Liu et al. [12] were not complete genome sequences and were not marked with the relevant symptom onset information. Hence they cannot help to distinguish and/or find the potential sources of $\mathrm{A} / \mathrm{H} 3 \mathrm{~N} 2$ infection in the transmission tree. Conditional on the available information, the evidence about how co-infection alters the infectivity of A/H3N2 virus is lacking. Nevertheless, the conclusion about enhanced infectivity of $\mathrm{A} / \mathrm{H} 1 \mathrm{~N} 1$ within coinfection is not affected.

The original method of reconstructing the transmission tree by Hens et al. [14] relies on three assumptions: all cases are observed; all of them except the index case are infected by another observed case; and the generation interval distribution remains unchanged. To apply to such outbreaks as studied here involving two viruses, some assumptions have been strengthened. There must be two index cases that were singly infected with different viruses or one index case that was co-infected with the two viruses. There are more than one transmission processes. In our study example, there are five transmission processes: $\mathrm{A} / \mathrm{H} 1 \mathrm{~N} 1$ to $\mathrm{A} / \mathrm{H} 1 \mathrm{~N} 1 ; \mathrm{A} / \mathrm{H} 3 \mathrm{~N} 2$ to $\mathrm{A} /$ $\mathrm{H} 3 \mathrm{~N} 2$; co-infection to $\mathrm{A} / \mathrm{H} 1 \mathrm{~N} 1$; co-infection to $\mathrm{A} / \mathrm{H} 3 \mathrm{~N} 2$ and co-infection to co-infection. Unfortunately the available data does not provide direct information for all the transmission processes, and it is not directly possible to assess the heterogeneity in generation intervals among transmission processes. In view of the similar estimates for generation intervals of different transmission processes [15], the generation interval distribution has been assumed to be unchanged over different types of infection as well as over the course of the outbreak.

Another aspect is how co-infection is generated. It could be due to an infection with one virus becoming a co-infection, or it could be a consequence of a cotransmission. And it is possible that going from A/H1N1 to co-infection is easier than going from $\mathrm{A} / \mathrm{H} 3 \mathrm{~N} 2$ to coinfection, or vice versa. Though this is an interesting issue [19] the limited information that we can collect from Liu et al. [12] cannot allow us to detect the order in which the two viruses are acquired by an co-infected individual and therefore no way to investigate the effect of the order in which the two viruses are acquired.

\section{Conclusion}

Reconstructing transmission trees provides useful information about generation interval and transmission rate of infectious diseases, which are important for designing containment strategies. In this study, the method of reconstructing the plausible transmission tree from the incomplete data of an outbreak caused by one virus (Hens et al. [14]) has been extended to the outbreak caused by two influenza A viruses. Our estimates of epidemiological characteristics such as the generation interval and the transmission rate of influenza A virus are well within the ranges estimated by others $[15,17]$. Our estimation shows that although co-infection with $\mathrm{A} / \mathrm{H} 1 \mathrm{~N} 1$ and $\mathrm{A} / \mathrm{H} 3 \mathrm{~N} 2$ viruses cannot be sustained by co-transmission, it enhances the single transmission of both viruses. However, the concluded enhancement of $\mathrm{A} / \mathrm{H} 3 \mathrm{~N} 2$ virus infectivity within 
co-infection should be taken with caution owing to the unknown infection source of seasonal $\mathrm{H} 3 \mathrm{~N} 2$ virus.

Cross-immunity, which characterises the interaction between different viruses when one virus re-infects individuals recovered from previous infection with another virus, has been well recognized and measured. Due to their relative rareness, co-infection and interactions between viruses within co-infections have not yet attracted the attention they deserve. To our knowledge this is the first analysis that estimates the interactions between influenza A viruses within co-infection. Theoretical modelling illustrates their potential role in generating the recurrent epidemics and alternation of the dominant virus in seasonal influenza [20, 21]. Surely this urges more empirical studies to investigate this important issue of influenza and other infectious diseases caused by multiple strains.

\section{Additional file}

Additional file 1: Effect of an (unidentified) index case of $A / H 3 N 2$

infection in building 2. (DOCX $186 \mathrm{~kb}$ )

\section{Abbreviations \\ ILI: influenza like illness; MLE: maximum likely estimate; SD: standard deviation.}

\section{Competing interests}

The authors declare that they have no competing interests.

\section{Authors' contributions}

Conceived and designed the study: XSZ and DD; Performed the study: XSZ: wrote the paper: XSZ and DD. Both authors read and approved the final manuscript.

\section{Acknowledgements}

This research was funded by the Public Health England. The authors gratefully acknowledge the assistance of Tom Nichols and also express gratitude to Drs Niel Hens and Michael Worobey for their constructive and helpful comments.

\section{Author details}

${ }^{1}$ Centre for Infectious Disease Surveillance and Control, Public Health England, 61 Colindale Avenue, London NW9 5EQ, UK. ${ }^{2}$ Medical Research Council Centre for Outbreak Analysis and Modelling, Department of Infectious Disease Epidemiology, Imperial College School of Public Health, Norfolk Place, London W2 1PG, UK. ${ }^{3}$ Medical Research Council Biostatistics Unit, University Forvie Site, Robinson Way, Cambridge CB2 OSR, UK.

Received: 27 May 2015 Accepted: 22 January 2016

H.P.

\section{References}

1. Denoeud L, Turbelin C, Ansart S, Valleron A-J, Flahault A, Carrat F. Predicting pneumonia and influenza mortality from morbidity data. PLoS ONE. 2007; 2(5):e464. doi:10.1371/journal.pone.0000464.

2. Finkelman BS, Viboud C, Koelle K, Ferrari MJ, Bharti N, Grenfell BT. Global patterns in seasonal activity of influenza A/H3N2, A/H1N1, and B from 1997 to 2005: viral coexistence and latitudinal gradients. PLoS ONE. 2007;12: e1296. doi:10.1371/journal.pone.0001296.

3. Pajak B, Stefanska I, Lepek K, Donevski S, Romanowska M, Szeliga M, et al. Rapid differentiation of mixed influenza A/H1N1 virus infections with seasonal and pandemic variants by multitemperature single-stranded conformational polymorphism analysis. J Clin Microbiol. 2011;49:2216-21.
4. Nie S, Roth RB, Stiles J, Mikhlina A, Lu X, Tang YW, et al. Evaluation of Alere I influenza A \& B for rapid detection of influenza A and B. J Clin Microbiol. 2014;52:3339-44.

5. Sonoguchi T, Naito H, Hara M, Takeuchi Y, Fukumi H. Cross-subtype protection in humans during sequential, overlapping, and or concurrent epidemics caused by H3N2 and H1N1 influenza-viruses. J Infect Dis. 1985;151:81-8.

6. Ghedin E, Fitch A, Boyne A, Griesemer S, DePasse J, Bera J, et al. Mixed infection and the genesis of influenza virus diversity. J Virol. 2009;83:8832-42.

7. Perez DR, Sorrell E, Angel M, Ye J, Hickman D, Pena L et al. Fitness of pandemic H1N1 and seasonal influenza A viruses during co-infection. PLoS Current. 2009; doi:10.1371/currents.RRN1011.

8. Peacey M, Hall RJ, Sonnberg S. Pandemic (H1N1) 2009 and seasonal influenza A(H1N1) co-infection, New Zealand, 2009. Emerg Infect Dis. 2010;16:1618-20.

9. Lee N, Chan PKS, Lam W, Szeto CC, Hui DS. Co-infection with pandemic H1N1 and seasonal H3N2 influenza viruses. Ann Intern Med. 2010;152:618-9.

10. Myers UA, Kasper MA, Yasuda CY, Savuth C, Spiro DJ, Hallpin R, et al. Dual infection of novel influenza viruses $\mathrm{A} / \mathrm{H} 1 \mathrm{~N} 1$ and $\mathrm{A} / \mathrm{H} 3 \mathrm{~N} 2$ in a cluster of Cambodian patients. Am J Trop Mede Hyg. 2011;85:961-3.

11. Toda S, Okamoto R, Nishida T, Nakao T, Yoshikawa M, Suzuki E, et al. Isolation of influenza $\mathrm{A} / \mathrm{H} 3$ and $B$ viruses from an influenza patient: confirmation of co-infection by two influenza viruses. Jpn J Infect Dis. 2006;59:142-3.

12. Liu W, Li ZD, Tang F, Wei MT, Tong YG, Zhang L, et al. Mixed infections of pandemic $\mathrm{H} 1 \mathrm{~N} 1$ and seasonal H3N2 viruses in 1 outbreak. Clin Infect Dis. 2010;50:1359-65.

13. Wallinga J, Teunis P. Different epidemic curves for severe acute respiratory syndrome reveal similar impacts of control measures. Am J Epidemiol. 2004; 160(6):509-16

14. Hens N, Calatayud L, Kurkela S, Tamme T, Wallinga J. Robust reconstruction and analysis of outbreak data: influenza $A(\mathrm{H} 1 \mathrm{~N} 1) \mathrm{v}$ transmission in a schoolbased population. Am J Epidemiol. 2012;176:196-203.

15. Boëlle P-Y, Ansart S, Cori A, Valleron A-J. Transmission parameters of the A/ H1N1 (2009) influenza virus pandemic: a review. Influenza Other Respi Viruses. 2011;5:306-16

16. Nelder JA, Mead R. A simplex method for function minimization. Comput J. 1965:7:308-13.

17. Biggerstaff M, Cauchemez S, Reed C, Gambhrir M, Fineli L. Estimates of the reproduction number for seasonal, pandemic, and zoonotic influenza: a systematic review of the literature. BMC Infect Dis. 2014;14:480.

18. Jombart T, Cori A, Didelot X, Cauchemez S, Fraser C, Ferguson N. Bayesian Reconstruction of Disease Outbreaks by Combining Epidemiologic and Genomic Data. PLoS Comput Biol. 2014;10(1):e1003457. doi:10.1371/journal. pcbi. 1003457

19. Davis BM, Aiello AE, Dawid S, Rohani P, Shrestha S, Foxman B. Influenza and community-acquired pneumonia interactions: the impact of order and time of infection on population patterns. Am J Epidemiol. 2012;175:363-7.

20. Zhang X-S, Cao K-F. The impact of coinfections and their simultaneous transmission on antigenic diversity and epidemic cycling of infectious diseases. BioMed Res Intern. 2014; Article ID 375862. http://dx.doi.org/10. 1155/2014/375862.

21. Zhang X-S. Strain interactions as a mechanism for dominant strain alternation and incidence oscillation in infectious diseases: seasonal influenza as a case study. PLoS ONE. 2015;10(11):e0142170. doi:10.1371/ journalpone.0142170.
Submit your next manuscript to BioMed Central and we will help you at every step:

- We accept pre-submission inquiries

- Our selector tool helps you to find the most relevant journal

- We provide round the clock customer support

- Convenient online submission

- Thorough peer review

- Inclusion in PubMed and all major indexing services

- Maximum visibility for your research

Submit your manuscript at www.biomedcentral.com/submit 